\title{
THE QUANTUM-LIMITED COMB LINESHAPE FOR A MODE-LOCKED Ti:SAPPHIRE LASER
}

\author{
J. Willits, J. K. Wahlstrand, C. R. Menyuk, and S. T. Cundiff \\ JILA, National Institute of Standards and Technology, and the University of Colorado at Boulder \\ 440 UCB, Boulder, CO,
}

\begin{abstract}
$\underline{\text { Abstract }}$
We have calculated the quantum-limited lineshape of the comb lines from a mode-locked Ti:sapphire laser using experimentally-derived parameters for the linear response and quantum noise drivers from a generalized soliton perturbation theory. The detailed lineshape has small contributions from relaxation oscillations and frequency pulling. Our results are relevant in determining the fundamental limitation due to amplified spontaneous emission on the uncertainty of frequency measurements using femtosecond combs.
\end{abstract}

\section{$\underline{\text { Introduction }}$}

When light passes though any gain medium, it will acquire noise through the process of amplified spontaneous emission (ASE) [1]. The ASE noise sets a fundamental limit on the linewidth in lasers. For continuous wave lasers, this fundamental limit (the well-known Schawlow-Townes linewidth) is largely due to phase jitter [2]. Due to their intrinsic nonlinearity, the situation with mode-locked lasers is more complicated. A mode-locked laser produces train of regularly spaced pulses, whose frequency spectrum is a series of comb lines. The laser output is characterized by four pulse parameters: the pulse's energy, its central or carrier frequency, its central time, and its phase. In addition, the gain of the lasing medium is a dynamical parameter. The ASE noise from the gain medium perturbs all five parameters as the pulse circulates in the laser cavity. The properties of all the comb lines are determined by the evolution of the four pulse parameters from one round trip to the next. However, each parameter can depend on the others in a complicated and not easily calculated way.

Recently, we quantitatively measured the linear response to a perturbation of the pulse energy, the central frequency, the round-trip gain [3], the central time, and the phase [4] of a mode-locked Ti:sapphire laser. The principal motivation for that work was to be able to quantitatively predict the quantum-noise-limited power spectra of the laser comb lines. Here, we carry out that calculation, taking into account the effects of gain oscillations, frequency pulling, and the coupling of the pulse energy and central frequency fluctuations into timing and phase jitter.

\section{Linear response and noise drivers}

The linear response of the laser to perturbations is [5]

$$
\frac{d \mathbf{v}}{d t}=-\mathbf{A} \cdot \mathbf{v}+\mathbf{S}
$$

where $\mathbf{v}$ is a vector of the deviations of the five parameters from their equilibrium values, $\mathbf{S}$ is a vector of noise sources for each parameter, and $\mathbf{A}$ is a matrix of coefficients that describe the linear response of each parameter to changes in itself or the others. Given $\mathbf{A}$ and $\mathbf{S}$, one can calculate the timing and phase jitter [5], which leads to the optical frequency comb linewidth [6].

To calculate the noise spectrum, we use the formalism of stochastic differential equations [7]. The quantum noise drivers were derived using a generalized perturbation theory for Gaussian pulses in a dispersion managed soliton laser [3]. Using calculated values for the quantum noise drivers, D, along with measured values of the $\mathbf{A}$ matrix we can now use the equation

$\frac{d\left\langle\Delta v_{i} \Delta v_{j}\right\rangle}{d T}=\sum_{k, l}\left(A_{i k}+A_{j l}\right)\left\langle\Delta v_{k} \Delta v_{l}\right\rangle+D_{i j}$,

where $\left\langle S_{i}(T) S_{j}\left(T_{0}\right)\right\rangle=D_{i j} \delta\left(T-T_{0}\right) \quad$ relates the elements of the vector $\mathbf{S}$ to to the elements of the matrix $\mathrm{D}$, to solve for the second moments of the of laser dynamical variables.

\section{Comb lineshape}

Neglecting the direct effects of amplitude and central frequency noise, the power spectral density of one comb line is

$$
\begin{aligned}
& I(\Omega, \omega) \propto \int_{0}^{\infty} d T \cos (\omega T) \exp \left(-\frac{1}{2} \Omega^{2}\left\langle[\Delta \tau(T)]^{2}\right\rangle+\right. \\
& \left.\Omega\langle\Delta \tau(T) \Delta \theta(T)\rangle-\frac{1}{2}\left\langle[\Delta \theta(T)]^{2}\right\rangle\right)
\end{aligned}
$$

where $\Omega$ denotes the difference between the frequency of the comb line of interest and the central frequency $\omega_{\text {eq }}$, and $\omega$ is the frequency with respect to 
the center of that line. The spectrum consists of a Lorentzian part and a much smaller high frequency part at the relaxation oscilation frequency, around $400 \mathrm{kHz}$ for the laser we measured, that arises from the detailed pulse dynamics. The Lorentzian linewidth of three comb lines is shown in Fig. 1.

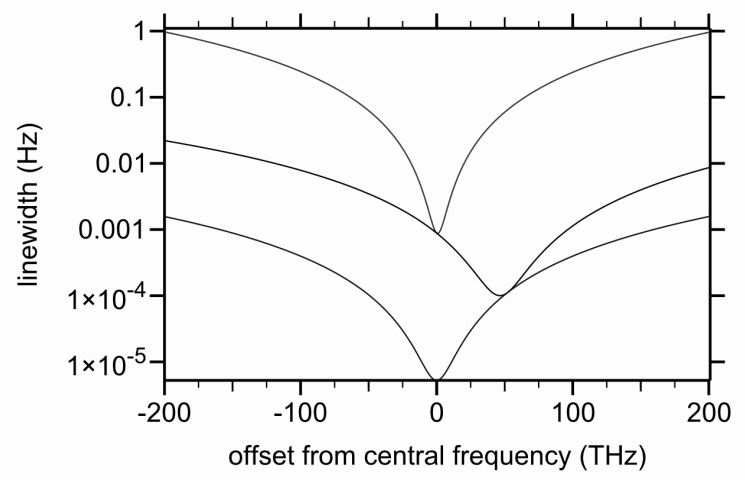

Fig. 1. Linewidth as a function of $\Omega / 2 \pi$ for the measured values and for various $\mathbf{A}$ parameters. The red curve is the result with the full set of parameters measured in [3] and [4]. The black curve is the result when timing jitter caused by dispersion is neglected. The blue curve is the result when, in addition, timing and phase jitter driven by intensity fluctuations is neglected.

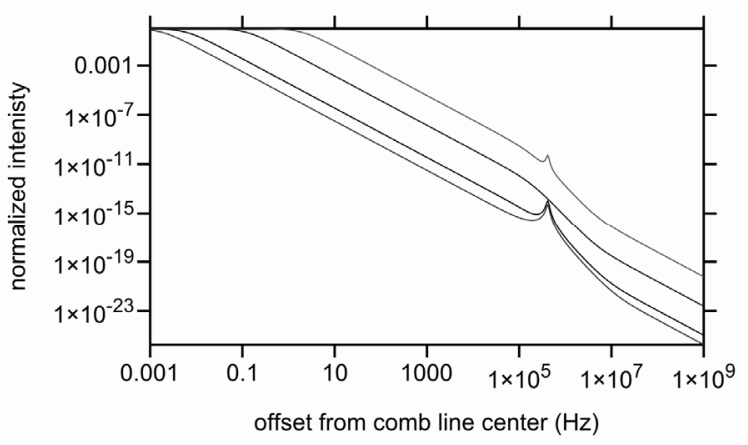

Fig. 2. Power spectrum of comb lines in various parts of the spectrum (red: $\Omega / 2 \pi=0 \mathrm{THz}$, black: $\Omega / 2 \pi=10 \mathrm{THz}$, blue: $\Omega / 2 \pi$ $=50 \mathrm{THz}$, green: $\Omega / 2 \pi=200 \mathrm{THz}$ ).

In Fig. 2, we show one half of the lineshape of the comb lines from a free-running laser in a few different parts of the spectrum, calculated using Eq. (3) for the particular laser studied in [3] and [4]. The line is, to a very good approximation, Lorentzian until $200 \mathrm{~dB}$ down from the peak, consistent with the results of Kärtner et al. [6], but when feedback is introduced (not shown), the details of the lineshape become relatively more important. The most notable feature is the peak at roughly $400 \mathrm{kHz}$, the relaxation oscillation frequency. The width of this peak depends on the strength of the effective saturable absorber that mode-locks the laser, which is strongly influenced by the pump power [3]. Note that as timing jitter becomes more important, the relaxation oscillation peak becomes relatively less important.
The exact shape of the spectrum depends on the relative strength of the intensity and central frequency contributions to the timing jitter.

\section{Conclusion}

While ASE noise is not currently the limiting factor in the linewidth for applications of femtosecond frequency combs [8], our approach could be used to calculate the effects of technical noise as well if one alters the driving terms contained in $\mathbf{D}$. We will also present results using a simple model for feedback to calculate the spectrum of a phase-locked comb, relevant to optical frequency metrology applications.

\section{References}

R. Loudon, Quantum Theory of Light. Clarendon. Oxford, UK, 2000.

A. L. Schawlow and C. H. Townes, "Infrared and Optical Masers," Phys. Rev. vol. 112, pp. 1940 (1958).

C. R. Menyuk, J. K. Wahlstrand, J. Willits, R. P. Smith, T. R. Schibli, and S. T. Cundiff, "Pulse dynamics in mode-locked lasers: relaxation oscillations and frequency pulling," Opt. Express vol. 15, pp. 66776689 (2007).

[4] J. K. Wahlstrand, J. T. Willits, T. R. Schibli, C. R. Menyuk, and S. T. Cundiff, "Quantitative measurement of timing and phase dynamics in a mode-locked laser," Opt. Lett. vol. 32, pp. 3426-3428 (2007).

H. Haus and A. Mecozzi, "Noise of modelocked lasers," IEEE J. Quantum Electron. 29, pp. 983-996 (1993).

[6] F. X. Kärtner, U. Morgner, T. Schibli, R. Ell, H. A. Haus, J. G. Fujimoto, and E. P. Ippen, "Few-Cycle Pulses Directly from the Laser" in Few-cycle pulses and Applications, F. X. Kartner, ed. (Springer, Berlin, 2004).

[7] P. Wai and C. Menyuk, "Polarization mode dispersion, decorrelation, and diffusion in optical fibers with randomly varying birefringence," J. Lightwave Technology vol. 14, pp. 148-157 (1996). See the appendix.

[8] N. R. Newbury and W. C. Swann, "Lownoise fiber-laser frequency combs," J. Opt. Soc. Am. B vol. 24, pp. 1756-1770 (2007). 